\title{
Cortical Lewy Body Dementia
}

\author{
W. R. G. GIBB \\ University Department of Neurology, King's College Hospital, Denmark Hill, London SE5, UK \\ Correspondence: Department of Neurology, Institute of Psychiatry, De Crespigny Park, London \\ SE5 $8 A F$
}

\begin{abstract}
In cortical Lewy body dementia the distribution of Lewy bodies in the nervous system follows that of Parkinson's disease, except for their greater profusion in the cerebral cortex. The cortical tangles and plaques of Alzheimer pathology are often present, the likely explanation being that Alzheimer pathology provokes dementia in many patients. Pure cortical Lewy body dementia without Alzheimer pathology is uncommon. The age of onset reflects that of Parkinson's disease, and clinical features, though not diagnostic, include aphasias, apraxias, agnosias, paranoid delusions and visual hallucinations. Parkinsonism may present before or after the dementia, and survival duration is approximately half that seen in Parkinson's disease without dementia.
\end{abstract}

\section{Introduction}

Cortical Lewy body dementia refers to the association of moderate to severe dementia and large numbers of cortical Lewy bodies. In addition there are always Lewy bodies in other regions of the nervous system, most notably the substantia nigra, and Parkinson's disease (PD) is often present.

There are two reasons why there is difficulty defining the disorder both clinically and pathologically. These concern its relationship to PD and to Alzheimer's disease (AD). In PD it is not generally recognized that Lewy bodies occur in the cerebral cortex, so their presence here has suggested that the disorder is separate from the pathology of PD. Furthermore PD does not always occur with the dementia. Cortical Alzheimer pathology is usually present and according to some authors this further divorces the condition from PD. It has also been proposed that the Alzheimer pathology is not really that of $\mathrm{AD}$, because some cases have a high frequency of plaques in preference to neurofibrillary tangles. Consequently the term diffuse Lewy body disease has been adopted in several publications to indicate that the combination of cortical pathologies identifies a unique disorder. There is little evidence to support this view.

\section{Relationship between Cortical Lewy Body Dementia and Parkinson's Disease}

The Lewy body is a filamentous inclusion present in the cytoplasm and processes of specific diseased nerve cells, indicating a particular kind of 
degenerative process. It is always associated with nerve cell degeneration, but often provides the most conspicuous evidence of early disease.

In PD and cortical Lewy body dementia Lewy bodies and nerve cell loss are found in the ventral tegmentum, locus coeruleus, thalamus, nucleus basalis of Meynert, raphe nuclei, and throughout the autonomic nervous system, as well as in the substantia nigra, limbic areas and cerebral cortex. Occasionally nerve cell loss in autonomic nuclei is sufficiently severe to be associated with moderate autonomic failure. Cell loss in the nucleus basalis, causing a loss of cortical choline acetyltransferase, is associated with dementia, but Lewy body degeneration in other areas has no agreed clinical correlate.

Another group of people to consider are those without PD or dementia who are found to have Lewy bodies in the substantia nigra and these other locations at the time of death. Their population prevalence rises from approximately $2 \%$ in the sixth decade, towards $10 \%$ in the ninth decade (Gibb and Lees, 1988a). They are referred to as having incidental Lewy body disease, but they correspond to a preclinical phase of $\mathrm{PD}$, which we know exists from biochemical data indicating that about $20 \%$ of the normal striatal dopamine remains when PD symptoms first appear. They show progressive nigral degeneration, but the nigral cell counts are below the approximate threshold of $40 \%$ of normal which coincides with the onset of symptoms of $\mathrm{PD}$.

These conditions (PD, dementia, autonomic failure, incidental disease) form part of the same pathological disorder called idiopathic Lewy body disease, with overlapping clinical syndromes reflecting the site of emphasis of pathology.

Traditionally the cerebral cortex has not been carefully examined in PD, because this diagnosis can be satisfactorily established by examination of the basal ganglia. In recent years the pathological basis for dementia in PD has come under scrutiny. Okazaki and colleagues (1961) first described cortical Lewy bodies in two patients with dementia and PD, although Helfand (1935) found them many years before in a case of Hallervorden--Spatz disease, manifest by dementia and a parkinsonian disorder. The Japanese became especially aware of the occurrence of Lewy bodies in the cerebral cortex in PD with dementia (Gibb et al., 1987), and Forno described them in both incidental Lewy body disease (1969) and PD without dementia (1966, 1969). In one recent study of patients without dementia they were found in one section of medial temporal cortex in 30\% (Gibb and Lees, 1987), thus reflecting the lack of previous scrutiny of this area, through lack of necessity. Dickson et al. (1989) found that 33 of $44(75 \%)$ patients with varying degrees of dementia with or without PD had Lewy bodies, when at least one section of "hippocampus and parahippocampal gyrus" was examined. A few PD patients have moderate numbers of Lewy bodies with spread into remaining temporal and frontal cortex, and rare patients have extensive cortical involvement with spread to parietal and occipital regions. Thus extension of Lewy bodies into certain limbic areas and the cerebral cortex is part of the spectrum of PD and Lewy 'body disease. 


\section{Relationship between Cortical Lewy Body Dementia and Alzheimer's Disease}

Reports continue to appear in the literature describing patients with dementia, cortical Lewy bodies and the tangles and plaques of $\mathrm{AD}$, purporting to provide evidence for a greater than chance association between these pathological processes, although this argument cannot be justified by randomly describing such cases. Cortical pathology predominates in $\mathrm{AD}$ and brainstem pathology predominates in PD, but many of the same cerebral regions are involved in both diseases, causing identical neurochemical changes. Dementia is commonly linked with one or both pathologies in the nucleus basalis and cerebral cortex. If neurofibrillary tangles and Lewy bodies are frequently found together in dementia this does not necessarily mean that they have a greater than chance association, because of their additive effect. The possibility of a greater than chance association must be examined in a different way.

We examined the possibility of an association between AD and PD by comparing the age-specific prevalence of Lewy bodies in the substantia nigra in 121 cases of $\mathrm{AD}$ and 273 control subjects (Gibb et al., 1989a). Twenty-five of the Alzheimer cases over 60 showed Lewy bodies, but only 14 $(14.0 \%)$ of these had mild nigral cell loss compatible with presymptomatic PD. Counts of tangles and plaques in the cortex were lower in cases of AD with Lewy bodies, compared to those without, but cortical choline acetyltransferase levels were similar. This suggests that Lewy body degeneration in the nucleus basalis of Meynert contributes to the cortical deficit of acetylcholine, but not to the cortical Alzheimer pathology. At least three other studies have also found that patients having dementia with cortical Lewy bodies have lower cortical tangle, and, to a lesser extent, plaque counts compared with patients without Lewy bodies (Joachim et al., 1988; Bierer et al., 1990; Hansen et al., 1990). In one of these studies cortical choline acetyltransferase levels were lower in the subjects with cortical Lewy bodies (Hansen et al., 1990), which may be a reflection of combined Alzheimer and Lewy body pathology in the nucleus basalis.

Some groups have also reported patients with cortical Lewy body dementia with numerous plaques, but few tangles, suggesting the disorder is unlike AD (Dickson et al., 1989; Perry et al., 1990). However, many cases with tangles are described, and the predominance of plaques may represent plaque predominant $\mathrm{AD}$. Other studies have actively endeavoured to distinguish the condition from $\mathrm{AD}$. In one report of cases with cortical plaques, but few tangles, the plaques were less frequently associated with neurites containing paired helical filaments than in $\mathrm{AD}$ with cortical tangles (Dickson et al., 1989). There was no comparison with plaque only Alzheimer cases (without Lewy bodies), and the distinction became blurred in cases of "diffuse Lewy body disease" with cortical tangles. Indeed an article appearing in the same journal reports this observation in AD (Probst et al., 1989). In five other patients with diffuse Lewy body disease cortical and nigral Lewy bodies showed the same antigenic profile as previously reported 
in $\mathrm{PD}$, except for a reaction with tau antibody (Galloway et al., 1989). Relatively few cases have been examined with these antibodies, and experience with different antibodies has varied considerably, so the validity and relevance of the positive tau reaction is unclear. This result does not match the observation that extension of Lewy bodies to the neocortex forms a continuum with PD pathology. In another study one patient with numerous cortical Lewy bodies, among five other cases of Lewy body disease, failed to show tau immunoreactive Lewy bodies (Bancher et al., 1989). Thus, there remains no evidence for a qualitative difference between cortical Lewy body dementia and either PD or AD.

\section{To What Extent Does Cortical Lewy Body Dementia Relate to Dementia in PD?}

The three main pathological lesions under consideration as potential causes of dementia in PD are cortical Lewy bodies, Alzheimer pathology, and Lewy body degeneration in the nucleus basalis (Gibb, 1989). Three recent studies have examined the clinical features of patients developing PD before age 40 or 46 years, and with mean or median survivals of 12 to 19 years (Lima et al., 1987; Quinn et al., 1987; Gibb and Lees, 1988b). There were 123 patients in all, and at the time of the studies nine were aged over 65 years. Only one of the 123 patients $(0.8 \%)$ had developed dementia despite their long duration of survival with PD. In contrast, a number of studies show that older patients with PD develop dementia at over twice the rate of the normal population, and often in disease of short duration (Brown and Marsden, 1984). These data imply that dementia in PD is more closely related to age, than to duration of the disorder.

Rare patients with dementia and Lewy bodies in the cerebral cortex, as the only pathological finding at this site, have presented before the age of 40 years (Gibb et al., 1987). As yet, there is no evidence that the chance of spread of Lewy bodies into the limbic regions and cerebral cortex increases with age. The implication of the low incidence of dementia in the 123 young onset patients is that cortical Lewy body disease of sufficient severity to cause dementia, in the absence of Alzheimer pathology, occurs at approximately this frequency (i.e. $0 \cdot 8 \%$ ) because Alzheimer pathology would be uncommon at this age.

Although cortical Lewy bodies do not appear to be an age-related phenomenon the frequency of cortical Alzheimer pathology does increase with age. Data abstracted from seven studies show that the prevalence of any neurofibrillary tangles in the hippocampus of non-demented persons is $8 \%$ in the $40-49$ year group, $40 \%$ in the $50-59$ year group, $50 \%$ in the $60-$ 69 year group, $57 \%$ in the $70-79$ year group, $66 \%$ in the $80-89$ year group, and $72 \%$ in the $90-99$ year group (Gibb, 1989). The addition of this Alzheimer pathology to cortical Lewy bodies means that the combination of pathologies is likely to account for dementia in another few percent (perhaps $5-8 \%$ ) of PD patients. 
Thus, in most cases of moderate and severe dementia in PD Alzheimer pathology provides a major contribution, with Lewy bodies in the cerebral cortex and nucleus basalis providing important additional contributions. Indeed in some reports, cortical Lewy bodies have been sparse, and the Alzheimer pathology has been more obviously linked to the dementia (Dickson et al., 1989). Patients with dementia and cortical Lewy bodies with very little or no Alzheimer pathology are uncommon.

Evidently this is not a new disorder, but because there is no definition more rigid than the occurrence of dementia and cortical Lewy bodies, the terms cortical Lewy body dementia or diffuse Lewy body disease are used very loosely. These patients are now frequently described in the literature, but were it not for the coincidence of Alzheimer pathology very few would have dementia. There is insufficient evidence to be persuaded that a pathogenetically distinct disorder exists (Hansen et al., 1990; Perry et al., 1990).

\section{Clinical Features}

Patients with cortical Lewy body dementia have been reported with symptom onset ranging from age 26 to 74 years, with a mean of 58 years, thus following the age scatter of PD. In $20 \%$ of reports dementia occurred in isolation, in 30\% dementia occurred first and PD second, in $32 \%$ dementia and PD developed together, and in 18\% PD presented first and dementia second. Cortical features such as aphasia, apraxia, agnosia and topographical disorientation are common, as in cortical pathologies such as AD. Paranoid delusions, visual hallucinations and fluctuating confusion are also common, as in patients with PD. It is argued that delusions and hallucinations are diagnostic of the condition, but these symptoms are reported in about $15 \%$ of patients believed to have $\mathrm{AD}$ alone, confirmed by pathological examination in a few. Average life expectancy is approximately half that of PD at 8 years.

Of the half dozen patients reported with no trace of $\mathrm{AD}$ in the cortex one example was a 27-year-old woman, who presented in 1961 with lethargy and irritability, insomnia and paranoid delusions (Gibb et al., 1987). She developed a rest tremor and rigidity of the right arm and leg. Two years from onset her sleep rhythm was disturbed, her walking was slow and her balance poor. She lay in bed with eyes closed, talking in a high pitched monotonous voice. There was palilalia, echolalia, nominal and mild expressive dysphasias. Memory for recent events was poor and learning was impaired. Her verbal WAIS IQ was 91 and her performance IQ was 74 . The verbal test scores were estimated to be well below her premorbid level. She easily became agitated, when a pronounced rest tremor developed in both hands. There was generalized poverty of movement with impairment of the ability to perform rapid alternating movements in the limbs, and tone was increased with cogwheel rigidity.

She was given anticholinergic drugs and 6 months later readmitted to hospital because of paranoid delusions and visual and auditory hallucina- 
tions. At times she sat with her eyes closed and was apparently difficult to rouse, on other occasions she was affable and responded to commands. Her IQ had deteriorated. Bradykinesia and rigidity were marked, and there was a coarse tremor. She died at the age of 30 , after an illness lasting 3 years. The CT scan showed cerebral atrophy.

\section{Pathological Features}

Lewy bodies and nerve cell loss are found in the parahippocampus, cingulate gyrus, insular cortex, frontal and temporal cortex, and often in the parietal and occipital cortex. Only rarely are isolated inclusions present in the hippocampus, although in one exception Lewy bodies were numerous at this site (Tiller-Borcich and Forno, 1988).

Many cortical nerve cells may contain Lewy bodies, but the degree of cell loss is milder than in AD. This pathology is also present in the substantia nigra, locus coeruleus and other specific locations in the nervous system. In about half of all reported cases substantial Alzheimer cortical pathology has been described, usually moderate to large numbers of tangles and plaques, or predominantly plaques.

The Lewy bodies mainly occur in pyramidal cells in middle and deep (third, fifth and sixth) cortical layers. They are small, round, curved or triangular in shape. They have a filamentous structure, and contain neurofilament subunits, tubulin and microtubule associated proteins 1 and 2 (Galloway et al., 1988). Thus, they are derived from the neuronal cytoskeleton, but their exact origin is open to speculation. The precise structural arrangement of the Lewy body varies depending on its specific location in the nervous system. One such feature of cortical Lewy bodies is that they rarely contain dense components, such as cores and laminae, which represent dense packing of filament.

The standard stain for Lewy bodies is haematoxylin and eosin $(\mathrm{H} \& \mathrm{E})$, and this is sufficient for the demonstration of multiple cortical Lewy bodies. However, many of these bodies stain partially or not at all with $\mathrm{H} \& \mathrm{E}$, and are more clearly demonstrated by sensitive silver techniques, such as those based on the Bielschowsky method, or by an antibody to ubiquitin (Lennox et al., 1989).

\section{Differential Diagnosis}

Cortical dementias other than $\mathrm{AD}$, with or without Lewy bodies, are uncommon. Faced with such a patient it is not possible to accurately predict whether they have $\mathrm{AD}$, Alzheimer pathology with Lewy bodies predominantly subcortically, or Alzheimer pathology with subcortical and cortical Lewy bodies. In this setting cortical Lewy bodies without Alzheimer pathology is such an uncommon finding that it (pure cortical Lewy body dementia) deserves as much consideration as other sporadic cortical demen- 
tias. Nevertheless the occurrence of visual hallucinations, delusions, and fluctuating mental performance may suggest Lewy body disease. In this regard it is interesting to note that among patients with cortical Lewy body dementia (and Alzheimer pathology) those with visual hallucinations have lower levels of choline acetyltransferase in temporal and parietal cortex, compared with those patients without hallucinations (Perry et al., 1990). Thus, the lower choline acetyltransferase level in cases of dementia, Alzheimer pathology, and cortical Lewy bodies, in comparison with those cases having no Lewy bodies (Hansen et al., 1990), may fit with different frequencies of hallucinations.

The very uncommon situation of $\mathrm{AD}$ alone being associated with a parkinsonian disorder (Gibb et al., 1989b), points to the presence of brainstem Lewy bodies in both demented parkinsonians and parkinsonian dements. Most of these will have at least one or two Lewy bodies in limbic areas.

\section{Conclusion}

The term cortical Lewy body dementia suggests patients with a cortical dementia and cortical Lewy bodies (in profusion) without other significant cortical disease, but such patients are rare. However, there is a large group of patients now reported under this, or analogous titles, with cortical Alzheimer pathology and fewer Lewy bodies. In most of these cases the Alzheimer pathology plays a significant part in the cause of the dementia, and in this sense cortical Lewy body dementia is a misnomer. In other words the term cortical Lewy body dementia is used imprecisely, largely because it is difficult to know where to draw a line when the cortical spread of Lewy bodies (and for that matter Alzheimer pathology) varies substantially. Despite other recent protestations the spread of Lewy bodies into the cortex merges into a spectrum with $\mathrm{PD}$, and there is no firm evidence for a distinct disease, just the concurrence of two different disease processes-Alzheimer pathology and Lewy bodies.

\section{References}

Bancher, C., Lassman, Budka, H. et al. (1989). An antigenic profile of Lewy bodies: immunocytochemical indication for protein phosphorylation and ubiquitination. Fournal of Neuropathology and Experimental Neurology, 48, 81-93.

Bierer, L. M., Perl, D. P., Haroutunian, V., Mohs, R. G. and Davis, K. L. (1990). Neurofibrillary tangles, Alzheimer's disease, and Lewy bodies. Lanciet, i, 163.

Brown, R. G. and Marsden, C. D. (1984) How common is dementia in Parkinson's disease? Lancet, ii, 1262-1265.

Dickson, D. W., Crystal, H., Mattiace, L. A., Kress, Y., Schwagerl, A., Ksizak-Reding, H., Davies, P. and Yen, S. H. C. (1989). Diffuse Lewy body disease: light and electron microscopic immunocytochemistry of senile plaques. Acta Neuropathologica, 78, 572-584.

Forno, L. S. (1966). Pathology of parkinsonism: a preliminary report of 24 cases. Journal of Neurosurgery, 24, 266-271.

Forno, L. S. (1969). Concentric hyalin intraneuronal inclusions of Lewy type in the brains of 
elderly persons (50 incidental cases): relationship to parkinsonism. Journal of the American Geriatrics Society, 17, 557-575.

Galloway, P. G., Grundke-Iqbal, I., Iqbal, K. and Perry G. (1988). Lewy bodies contain epitopes both shared and distinct from Alzheimer neurofibrillary tangles. Fournal of Neuropathology and Experimental Neurology, 47, 654-663.

Galloway, P. G., Bergeron, C. and Perry, G. (1989). The presence of tau distinguishes Lewy bodies of diffuse Lewy body disease from those of idiopathic Parkinson's disease. Neuroscience Letters, 100, 6-10.

Gibb, W. R. G. (1989). Dementia and Parkinson's Disease. British Journal of Psychiatry, 154, 596-614.

Gibb, W. R. G. and Lees, A. J. (1987). Dementia in Parkinson's disease. Lancet, i, 861.

Gibb, W. R. G. and Lees, A. J. (1988a). The relevance of the Lewy body to the pathogenesis of idiopathic Parkinson's disease. Fournal of Neurology, Neurosurgery and Psychiatry, 51, 745752.

Gibb, W. R. G. and Lees, A. J. (1988b). A comparison of clinical and pathological features of young and old-onset Parkinson's disease. Neurology, 38, 1402-1406.

Gibb, W. R. G., Esiri, M. M. and Lees, A. J. (1987). Clinical and pathological features of diffuse cortical Lewy body disease (Lewy body dementia). Brain, 110, 1131-1153.

Gibb, W. R. G., Mountjoy, C. Q., Mann, D. M. A. and Lees, A. J. (1989a). A pathological study of the association between Lewy body disease and Alzheimer's disease. Fournal of Neurology, Neurosurgery and Psychiatry, 52, 701-708.

Gibb, W. R. G., Mountjoy, C. Q., Mann, D. M. A. and Lees, A. J. (1989b). The substantia nigra and ventral tegmental area in Alzheimer's disease and Down's Syndrome. Fournal of Neurology, Neurosurgery and Psychiatry, 52, 193-200.

Hansen, L., Salman, D., Galasko, D. et al. (1990). The Lewy body variant of Alzheimer's disease: a clinical and pathologic entity. Neurology, 40, 1-8.

Helfand, M. (1939). Status pigmentatus: its pathology and its relation to HallervordenSpatz disease. Fournal of Nervous and Mental Disease, 81, 662-675.

Joachim, C. L., Morris, J. H. and Selkoe, D. J. (1988). Clinically diagnosed Alzheimer's disease: autopsy results in 150 cases. Annals of Neurology, 24, 50-56.

Lennox, G., Love, J., Morrell, K., Landon, M. and Mayer R. J. (1989). Anti-ubiquitin immunocytochemistry is more sensitive than conventional techniques in the detection of diffuse Lewy body disease. Fournal of Neurology, Neurosurgery and Psychiatry, 52, 67-71.

Lima, B., Neves, G. and Nora, M. (1987). Juvenile parkinsonism: clinical and metabolic characteristics. Fournal of Neurology, Neurosurgery and Psychiatry, 50, 345-348.

Okazaki, H., Lipkin, L. E. and Aronson, S. M. (1961). Diffuse intracytoplasmic ganglionic inclusions (Lewy type, associated with progressive dementia and quadriparesis in flexion. Fournal of Neuropathology and Experimental Neurology, 20, 237-244.

Perry, R. H., Irving, D., Blessed, G., Fairbairn, A. and Perry, E. K. (1990). Senile dementia of Lewy body type. A clinically and neuropathologically distinct form of Lewy body dementia in the elderly. Fournal of the Neurological Sciences, 95, 119-139.

Probst, A., Anderton, B. H., Brion, J-H. and Ulrich, J. (1989). Senile plaque neurites fail to demonstrate anti-paired helical-filament and anti-microtubule-associated protein-tau immunoreactive proteins in the absence of neurofibrillary tangles in the neocortex. Acta Neuropathologica, 77, 430-436.

Quinn, N. P., Critchley, P. and Marsden, C. D. (1987). Young onset Parkinson's disease. Movement Disorders, 2, 73-91.

Tiller-Borcich, J. K. and Forno, L. S. (1988). Parkinson's disease and dementia with neuronal inclusions in the cerebral cortex: Lewy bodies or Pick bodies. Fournal of Neuropathology and Experimental Neurology, 47, 526-535. 


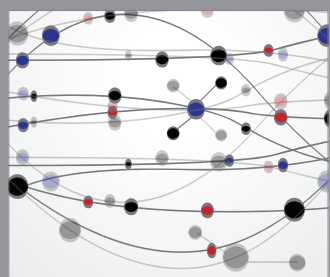

The Scientific World Journal
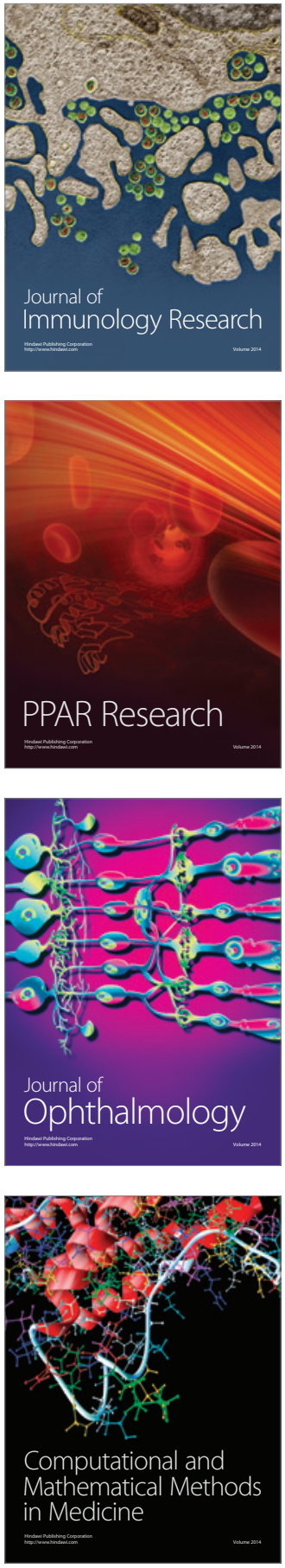

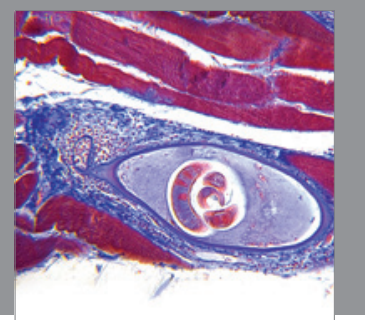

Gastroenterology

Research and Practice
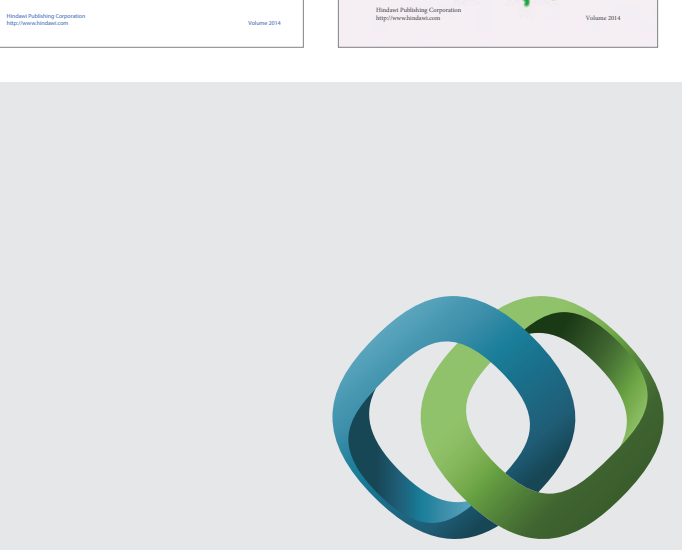

\section{Hindawi}

Submit your manuscripts at

http://www.hindawi.com
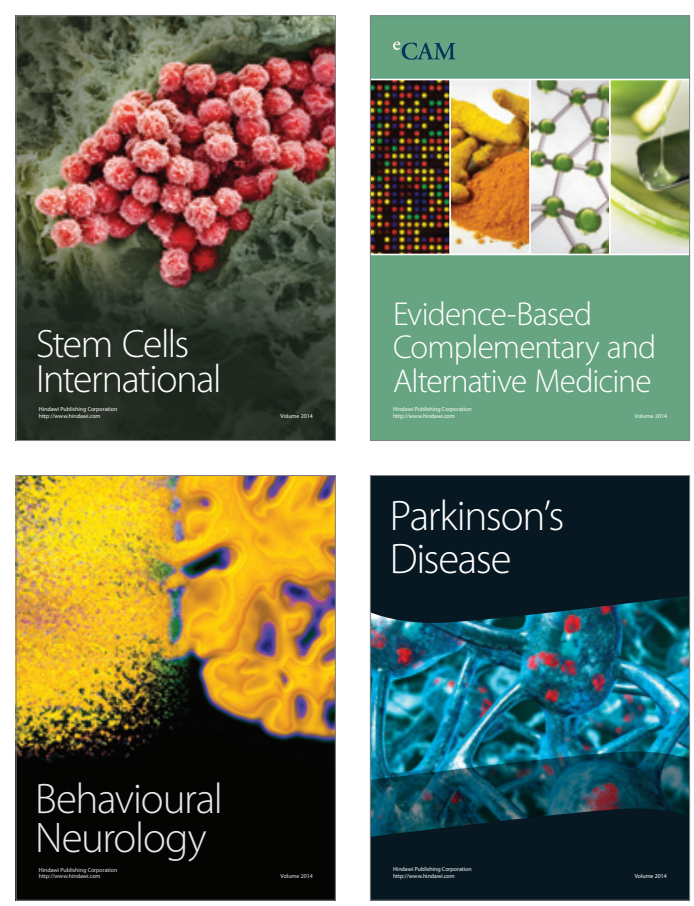

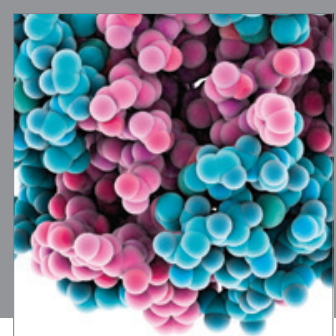

Journal of
Diabetes Research

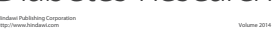

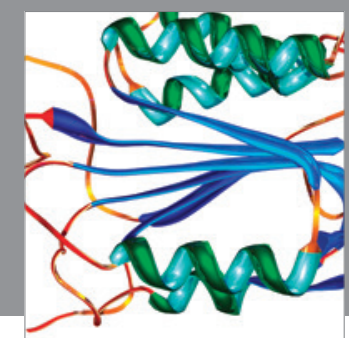

Disease Markers
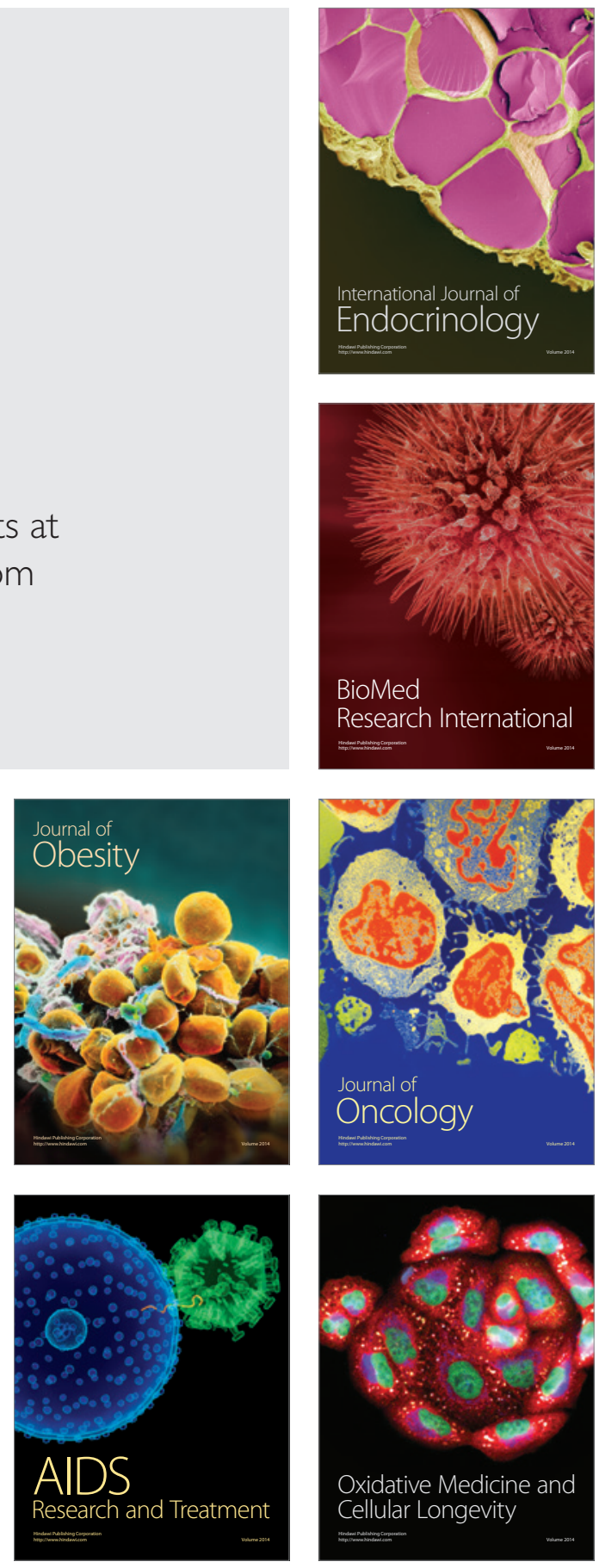